\title{
The relationship between leader emotional intelligence and psychological climate: An exploratory study
}

\author{
C. Klem \\ Department of Industrial Psychology, University of Stellenbosch \\ Stellenbosch 7600, Republic of South Africa \\ A.F. Schlechter* \\ School of Management Studies, University of Cape Town, \\ Private Bag, Rondebosch 7701, Republic of South Africa \\ Anton.Schlechter@uct.ac.za
}

Received March 2008

\begin{abstract}
This study, which was conducted in a clothing manufacturing plant, investigated the relationship between two important constructs for organisations, namely: leader emotional intelligence and psychological climate. A random cluster sample of 600 participants were drawn from a total employee population of 1725 and 297 completed responses were returned for analyses (49,5\% response rate). An Exploratory Factor Analysis (EFA) was conducted on both the Swinburne University Emotional Intelligence Test (SUEIT) and the Organisational Climate Questionnaire of Koys and DeCotiis (1991). Both scales were found to be valid and reliable. Stepwise Discriminant analysis provided evidence that a single psychological climate existed in the organisation. The results of a Pearson correlation analysis and Hierarchical Multiple Regression further indicated that leader emotional intelligence is significantly positively related to the psychological climate.
\end{abstract}

*To whom all correspondence should be addressed.

\section{Introduction}

What makes a successful organisation? The increasingly competitive business environment has prompted organisations, management-researchers and practitioners alike to search for new and innovative answers to this important question. De Vries (2001) found that successful organisations share several important characteristics. One of these characteristics is an organisational climate that fosters and nurtures high employee motivation and morale. De Vries (2001) provided evidence that organisational climate has a significant impact on organisational performance. Similarly, Goleman (2002) proposes that organisational climate can account for twenty to thirty percent of an organisation's success.

If organisational climate drives such a significant portion of business results, the question can rightly be asked, "What drives organisational climate?" Between 53 to 72 percent of how employees perceive their organisation's climate can be linked to the actions of one person - the leader (Kelner, Rivers \& O' Connell, 1996). According to Scholtz (2002) various studies have shown that the philosophy, policies and actions of the leader has a significant influence on the wellbeing of employees, the organisational culture and the organisational climate. Goleman (2002) suggests that leaders play a key role in driving the organisational climate and that their actions and behaviour, which are a result of their own emotional states, affect how the people they lead feel and perform. Business performance will, therefore, be influenced by how well leaders manage their own and their subordinate's moods. This ability to manage your own moods and that of others, is what Goleman (2002) defines as
Emotional Intelligence (EI). Although Goleman (2002) suggests that the emotionally intelligent leader has an effect on the organisational climate, no empirical studies could be found to validate this claim.

The specific objective of the present study was to investigate the relationship between the leaders' emotional intelligence and organisational climate, more specifically, the psychological climate of the organisation. This relationship is important, as it is believed that creating the right psychological climate will result in the effective utilisation of human capital in a manner that maximises the contribution of employees to the success of the organisation. The study is of an exploratory nature as no previously conducted research on the relationship between these two constructs could be found in the literature. Cilliers and Kossuth (2002) also suggested that future research on organisational climate should include more salutogenic constructs, with specific reference to emotional intelligence. This study attempts to address this gap in the literature.

\section{Emotional intelligence}

In recent years several comprehensive models of emotional intelligence provided alternative theoretical frameworks for conceptualising this construct. Although these models do not necessarily contradict one another, they represent different perspectives (Schutte, Malouff, Hall, Haggerty, Cooper, Golden \& Dornheim, 1998).

Emotional intelligence has its origin in the concept of 'social intelligence' which was first identified by Thorndike in 1920. Thorndike (1920) defined social intelligence as “... 
the ability to understand and manage men and women, boys and girls - to act wisely in human relations" (Walker \& Foley, 1973: 840). Gardner (1983) described intelligence to be a multifaceted attribute and differentiated between seven types of intelligence, namely: 1) spatial, 2) physical, 3) musical, 4) linguistic, 5) logical-mathematical, 6) interpersonal and 7) intrapersonal intelligence. Social intelligence can be taken to consist of a person's interpersonal and intrapersonal intelligence. Intrapersonal intelligence relates to one's intelligence in dealing with oneself. It is the ability to symbolize complex and highly differentiated sets of feelings (Gardner, 1983). Interpersonal intelligence relates to one's intelligence in dealing with others. It is the ability to notice and make distinctions among other individuals, and in particular, among their moods, temperaments, motivations and intentions (Gardner, 1983). Even though Gardner did not use the term emotional intelligence, his concepts of intrapersonal and interpersonal intelligence provided a foundation for later models of emotional intelligence (Schutte et al., 1998).

Several attempts have been made to conceptualise emotional intelligence. However, much of the current writing on this construct has approached emotional intelligence from a more popular angle often quoting anecdotal facts to support claims made by the author/s. This has not always been accompanied by empirical studies, particularly focusing on the measurement of the construct. The implications of this became apparent when the first attempts were made to measure the construct. It was found that the sampling domain for emotional intelligence was not clear-cut and its operationalisation strongly depended on the method of measurement (Petrides \& Furnham, 2000b). As a result, Petrides and Furnham (2000a) proposed a differentiation between trait and information-processing approaches to emotional intelligence, based on the different measurement approaches and operational definitions adopted by the various theorists. Trait emotional intelligence is embedded within the personality framework and is assessed through validated self-report inventories that measure typical behaviour. Information-processing emotional intelligence is specifically concerned with actual abilities, thus best assessed by means of maximum-performance measures consisting of items with correct or incorrect responses. Mayer, Salovey and Caruso (2000) also note the importance of understanding the various ways the term emotional intelligence is used by exploring emotional intelligence as a personality trait and as an ability. They, however, add a third meaning, namely emotional intelligence as zeitgeist, or a cultural trend.

\section{Emotional intelligence as a personality trait}

Some researchers, when defining emotional intelligence, refer to a long list of traits, attributes or abilities drawn from several aspects of personality. One such conceptualisation is that of Goleman (1995). He proposed that emotional intelligence consists of five dimensions: 1) self-awareness, 2) self-regulation, 3) motivation, 4) empathy and 5) social skills. He further describes the five dimensions of emotional intelligence in terms of twenty-five different emotional competencies. More recently Goleman (1998) defined emotional intelligence as "...the capacity for recognising our own feelings and those of others, for motivating ourselves, and for managing emotions well in ourselves and in our relationships" (Goleman, 1998: 317).

Bar-On's (1997) definition of EI proposed that one's ability to succeed in coping with environmental pressures is influenced by non-cognitive capabilities, competencies and skills. He developed the Bar-On Emotional Quotient Inventory (Bar-On, 1997) and conceptualises emotional intelligence as five broad areas of skills or competencies namely: 1) intrapersonal, 2) interpersonal, 3) adaptability, 4) stress management and 5) general mood.

Cooper and Sawaf (1997) formulated a model of emotional intelligence that relates specific skills and tendencies to what they called the Four Cornerstone model. This model moves emotional intelligence out of the realm of psychological analysis and philosophical theories, into the realm of direct knowing, exploration and application. The four dimensions are: 1) emotional literacy, 2) emotional fitness, 3) emotional depth, and 4) emotional alchemy. Cooper and Sawaf (1997) define emotional intelligence as “...the ability to sense, understand, and effectively apply the power and acumen of emotions as a source of human energy, information, connection, and influence" (Cooper \& Sawaf, 1997: xiii).

\section{Emotional intelligence as a mental ability}

From a mental ability perspective, emotional intelligence is conceptualised as mental abilities, skills, or capacities (i.e. much like EQ). Salovey and Mayer (1990) were among the first to propose the name emotional intelligence to define the ability of people to deal with their emotions as "...the subset of social intelligence that involves the ability to monitor one's own and others' feelings and emotions, to discriminate among them and to use this information to guide one's thinking and actions" (Salovey \& Mayer, 1990: 189). Their original model postulated that emotional intelligence consisted of three categories of variables that traditionally belonged in areas outside intelligence, namely: 1) appraisal and expression of emotion, 2) regulation of emotion and 3) utilisation of emotions in solving problems. A revised model was proposed by Mayer and Salovey (1997) that redefined emotional intelligence emphasising the cognitive components as well as conceptualising emotional intelligence in terms of potential for intellectual and emotional growth. The revised model consists of four distinct dimensions:

1. Appraisal and expression of emotion in the self - that relates to the individual's ability to understand his/her own deep emotions and to be able to express these emotions naturally. Individuals rating high on this dimension will sense and acknowledge their emotions well before most people.

2. Appraisal and recognition of emotion in others - that relates to the ability of individuals to perceive and understand the emotions of people around them. These individuals are sensitive to the feelings and emotions of others as well as 'reading their minds'. 
3. Regulation of emotion in the self - that relates to the ability of individuals to regulate their emotions, which will enable a more rapid recovery from psychological distress.

4. Use of emotion to facilitate performance - that relates to the ability of individuals to make use of their emotions by directing them towards constructive activities and personal performance (Mayer \& Salovey, 1997).

\section{Measurement of emotional intelligence}

Although the interest in emotional intelligence has advanced theoretically, the assessment of the construct has not kept pace. Several attempts were made to devise measures to assess the construct. These attempts represented a number of non-scientific as well as a wide variety of scientific measurement scales (Schutte et al., 1998). As discussed above the content of these scales vary widely as a consequence of the different conceptualisations of the term emotional intelligence.

These measurement models can be divided into: 1) Ability Scales - e.g. the Multifactor Emotional Intelligence Scale (MEIS) (Mayer \& Salovey, 1997) which measures emotional intelligence as an intelligence per se, i.e. as it relates to processing information; 2) Self-report Scales - e.g. the BarOn EQ-I (Bar-On, 1997) and the EQ-Map (Cooper \& Sawaf, 1997) which are two examples of self-report scales where respondents are asked to indicate to what extent a series of descriptive statements describe (or do not describe) themselves; and 3) Observer-rating Scales - e.g. the Emotional Competence Inventory (ECI) (Boyatzis, Goleman \& Hay/McBer, 1999) scale which is an example of a joint self-report/observer rating scale.

Therefore, in evaluating a measure of emotional intelligence, the content validity and the method by which the test gathers information, need to be considered (Mayer et al., 2000). According to Schutte et al. (1998) there is still a need for brief, validated measures of emotional intelligence that are based on a cohesive and comprehensive model of emotional intelligence.

\section{The psychological climate in the organisation}

A great deal of research and literature reviews of the organisational climate construct has appeared since the mid 1960's, illustrating the importance of the construct (Campbell, Dunnette, Lawler \& Weick, 1970; Forehand \& Gilmer, 1964; Glick, 1985; Hellriegel \& Slocum, 1974; James \& Jones, 1974; Joyce \& Slocum, 1982; Litwin \& Stringer, 1968). These reviews have been critical about the progress that has been made in the conceptualisation and measurement of organisational climate (Schneider \& Reichers, 1983). Considerable controversy still, however, remains around the construct, particularly regarding its measurement. Knowledge of organisational climate is important as it allows one to understand an individual's behaviour so that he or she can be managed effectively and efficiently (Tustin, 1993). Field and Abelson (1982) further regard the construct as important as it provides a conceptual link between analysis at the organisational and the individual level.

Many definitions of organisational climate exist (Forehand \& Gilmer, 1964; Hellriegel \& Slocum, 1974; Moran \& Volkwein, 1992; Ostroff \& Schmitt, 1993; West, Smith, Lu Feng \& Lawthom, 1998). However, the study of organisational climate is grounded in the theory of Kurt Lewin, who argued that behaviour is a function of the person and the environment. Based on this notion, Litwin and Stringer (1968:1) defined organisational climate as: "...a set of measurable properties of the work environment, perceived directly or indirectly by the people who live and work in this environment and assumed to influence their motivation and behaviour".

According to Glick (1985), the study of climate in organisations has been complicated by the fact that it is a complex, multi-level phenomenon. Initially, organisational climate was approached as an objective construct, consisting of organisational attributes such as structure, context and processes. It was believed that an accurate and objective measure of the degree to which these attributes was present in an organisation could be obtained, based on the assumption that these attributes affected the employees indirectly. The objective approach has been criticised and its validity questioned due to the fact that it ignored the individual's perceptions of organisational attributes (James \& Jones, 1974). The objective approach of organisational climate has been personified by Forehand and Gilmer's (1964) definition as: “ $\ldots$ the set of characteristics that describes an organisation and that (a) distinguish the organisation from other organisations, (b) are relatively enduring over time, and (c) influence the behaviour of people in the organisation" (Forehand \& Gilmer, 1964: $362)$.

In contrast, the perceptual approach believes that individuals are greatly influenced by their perceptions, or the psychological meaning they attach to organisational attributes. Schneider (1973) states that "...the concept of climate in the present research may best be described as personalistic; climate is an individual perception. There was no attempt to restrict the climate definition to perceptions shared by members of a work group or organisation" (Schneider, 1973: 254). Organisational climate is also defined by West et al. (1998) as the "...perceptions that organisation members share of fundamental elements of their organisation" (West et al., 1998: 262). Koys and DeCotiis (1991) suggest that organisational climate perceptions summarise the individual's description, rather than the evaluative reaction of his or her organisational experience; is relatively stable over time and are widely shared by the members of the organisational unit.

The controversy around organisational climate has been aggravated by the fact that the aggregated scores of the participating individuals in a climate survey, were considered to be an indication of the degree to which everyone in the organisation experienced a specific dimension in the climate. This operationalisation of organisational climate has been criticised by Guion (1973), questioning whether climate was organisational if the 
individual participants did not agree to what the organisational climate was. Individuals within the same organisation could view climate dimensions quite differently (Tustin, 1993).

The confusion as to whether organisational climate constituted an individual or an organisational attribute, caused by the emphasis placed on underlying psychological processes, led James and Jones (1974) to differentiate organisational climate from psychological climate. They recommend the term organisational climate when climate is regarded as an organisational attribute, and psychological climate, when climate is regarded as an individual attribute. Thus, psychological climate is studied at the individual level of analysis, referring to the individual's descriptions of organisational practices and procedures, while organisational climate is most often assessed through the average perceptions of the members of the organisation, thus referring to a collective description of the same environment (Joyce \& Slocum, 1982).

At an early stage Hellriegel and Slocum (1974) presented another aspect of climate at subsystem or group level. They stated: "Organisational climate refers to a set of attributes which can be perceived about a particular organisation and/or its subsystems, and that may be induced from the way that the organisation and/or its subsystems deal with their members and environment" (Hellriegel \& Slocum, 1974: 256). Joyce and Slocum (1982) clarify the controversy concerning organisational versus group versus individual climate. They state that all climates are perceptions that individuals have of their environment determined by the quasi-physical, quasi-social, quasiconceptual facts and intersubjectivity which an individual is aware of.

Climate has therefore evolved from being an exclusive organisational attribute to an attribute that may be subsystem specific (i.e. either the group or the individual). Although it has been defined at three levels of analysis in the organisation, the common elements are that climate has measurable, enduring qualities, which influence the behaviour of individuals in the organisation (Field \& Abelson, 1982). This present study focuses on the perceptions that individuals have of their environment and will therefore investigate psychological climate.

\section{Organisational climate versus organisational culture}

There has been considerable debate about the differences (and similarities) between organisational climate and organisational culture. No discussion of organisational climate will be complete without a reference to culture. Some researchers do not make a differentiation in terms of any frames of reference, but see the differences in terms of emphasis and degree, while other researchers believe that organisational climate is a totally different construct from culture. Sparrow and Gaston (1996) derived a number of conceptual differences from the literature based on their frames of organisational reference, units of theory and analysis, and implied level of awareness. Some of the differences between the two constructs are summarised in Table 1.

Table 1: Conceptual differences between organisational climate and culture

\begin{tabular}{|c|c|c|}
\hline Conceptual differences & Organisational climate & Organisational culture \\
\hline 1. Frames of organisational references & $\begin{array}{l}\text { Psychological schema: Based on hidden } \\
\text { personal values that may be aggregated } \\
\text { across organisations. }\end{array}$ & $\begin{array}{l}\text { Group understandings: Ways of } \\
\text { perceiving, thinking or feeling in relation } \\
\text { to a group's problems. }\end{array}$ \\
\hline 2. Unit of theory & $\begin{array}{l}\text { Individual and shared psychological } \\
\text { fields: Average of how people perceive the } \\
\text { way in which the environment is personally } \\
\text { beneficial or not. }\end{array}$ & $\begin{array}{l}\text { System-sanctioned behaviours: Norms, } \\
\text { beliefs and justifying ideologies that are } \\
\text { appropriate and beneficial to all members } \\
\text { of the system. }\end{array}$ \\
\hline 3. Unit of analysis & $\begin{array}{l}\text { Vary from individual to aggregate } \\
\text { measures of consensus at work group, } \\
\text { division or organisation level - shared and } \\
\text { learned perceptions resulting from policies, } \\
\text { practices and procedures. }\end{array}$ & $\begin{array}{l}\text { Always on collective groups, never the } \\
\text { individual - deeper elements of analysis } \\
\text { such as shared meanings, assumptions } \\
\text { and values that underlie these } \\
\text { organisational policies. }\end{array}$ \\
\hline 4. Implied level of consciousness & $\begin{array}{l}\text { Conscious subset of learned responses and } \\
\text { acquired meanings. }\end{array}$ & $\begin{array}{l}\text { Subconscious, taken-for-granted learned } \\
\text { responses. }\end{array}$ \\
\hline
\end{tabular}

Source: Sparrow et al. (1996)

Organisational culture researchers focus on qualitative analysis, often using interviews, case studies and observations. According to Schein (1989) organisational culture is visible in the organisation on three levels: 1) easy observable surface signals although they are difficult to interpret, e.g. language, symbols and myths; 2) reinforced behaviour patterns e.g. rituals, norms, beliefs and values and 3) deeper core values and assumptions which are perceived to be the most stable and contributing elements of organisational culture.

Organisational climate researchers on the other hand focus on quantitative measurement. The different facets of organisations and management styles, values and permitted behaviours are reflected by the numerical data obtained from these measurements. They measure those behavioural and value aspects of organisational culture that may be generalised across all organisations. Organisational climates are not necessarily the same across organisations; they are however, a collective set of constructs that are believed to be representative of life within most organisations. Organisational climate surveys are a popular tool that is used for Organisational Development (OD) initiatives, measuring the gaps of the existing and future desired organisational climate in an organisation (Sparrow \& Gaston, 1996). 


\section{Psychological climate dimensions}

As indicated in the above discussion, there appears to be little agreement on the dimensionality and thus the measurement of organisational climate. Although many dimensions have been identified, four common dimensions were originally suggested by Campbell et al. (1970), namely: 1) autonomy/control; 2) degree of structure; 3) rewards, and 4) consideration, warmth and support. It appears that these dimensions are consistent across all organisations. There may, however, be other dimensions that are organisation specific.

According to Cilliers and Kossuth (2002) organisational climate refers to the organisation's psychological atmosphere on the meta-level. On the operational level, organisational climate consists of organisational, interpersonal and individual dimensions. Table 2 is a summary of these various dimensions:

\section{The relationship between leader emotional intelligence and psychological climate}

Support provided by managers in their role as leaders is based on a positive, constructive and helpful attitude towards their subordinates in order to reach organisational goals. House, 1989 (cited in Cilliers \& Kossuth, 2002) conceptualises managerial support as information support, appraisal support, instrumental support and emotional support. The manner in which the above organisational climate dimensions are managed, as well as the quality of the leadership style of the manager, would therefore, influence the organisational climate.

Table 2: Dimensions of organisational climate

\begin{tabular}{l|l|l}
\hline \multicolumn{1}{c|}{ Organisational dimensions } & \multicolumn{1}{c}{$\begin{array}{c}\text { Interpersonal dimensions - Nature if } \\
\text { managerial support }\end{array}$} & \multicolumn{1}{c}{ Individual dimensions } \\
\hline $\begin{array}{l}\text { Formal level: } \\
\begin{array}{l}\text { Structure, policy, objectives, management } \\
\text { practice, task specialisation, decision } \\
\text { making, standard, and reward. }\end{array}\end{array}$ & $\begin{array}{l}\text { Directive properties: } \\
\text { Structure, role clarity, job standards, } \\
\text { managerial effectiveness and job } \\
\text { satisfaction. }\end{array}$ & $\begin{array}{l}\text { The individual's frame of reference } \\
\text { influences his/her perception of the nature of } \\
\text { organisational climate. }\end{array}$ \\
\hline $\begin{array}{l}\text { Informal level: } \\
\text { Identity, employee needs, responsibility, } \\
\text { interactive communication, information } \\
\text { sharing, support, warmth and conflict } \\
\text { handling. }\end{array}$ & $\begin{array}{l}\text { Interactive properties: } \\
\text { Communication, team functioning, } \\
\text { contribution to profits, conflict handling and } \\
\text { reward. }\end{array}$ & \\
\hline
\end{tabular}

Source: Cilliers Kossuth (2002)

The traditional organisational climate model in Field and Abelson (1982) indicates three main classes of variables that influence the perceived organisational climate: 1) external influences; 2) organisational influences; and 3) leadership/managerial influences. The awareness of these variables is moderated firstly by the group the individual is a member of, secondly the task of the individual, and thirdly the individual's personality. The leader influences the psychological climate by his/her managerial behaviour and leadership pattern, rewards and controls. According to Hughes, Ginnet and Curphy (2002) leadership involves both rational and emotional sides of human experience as people think, feel, hope and dream differently. Due to this fact, leaders use rational and/or emotional techniques to influence followers and have to weigh up the consequences of their actions. Leader emotional intelligence is necessary to distinguish which technique to use, to ensure that the desirable psychological climate is instilled in the follower. An adapted model of Field and Abelson (1982) that portrays this relationship is proposed in Figure 1.

\section{Aim of the study}

The aim or purpose of this study was to examine the relationship between the emotional intelligence of leaders/managers and psychological climate. In accordance with the proposed conceptual relationship between the constructs described above, the following two hypotheses were formulated:
Hypothesis 1: A single psychological climate profile exists in the organisation.

Hypothesis 2: A direct positive relationship exists between leader emotional intelligence and psychological climate.

\section{Method}

\section{Population and sample}

This research was done in a clothing manufacturing plant in the Western Cape with a total employee population of 1725 (Dept 1=238; Dept 2=403; Dept 3=481; Dept $4=482$ and Dept $5=121$ ). From this population a representative sample of 600 participants $(34,8 \%)$ were drawn, using proportional random cluster sampling. The sample consisted from 78 (13\%) employees from Department 1, 150 (25\%) employees from Department 2, 165 (27,5\%) employees from Department 3, 165 (27,5\%) employees from Department 4 and $42(7 \%)$ from Department 5.

Of the 600 questionnaires distributed, 297 (49.5\%) completed questionnaires were returned: 45 of Department 1, 61 of Department 2, 86 of Department 3, 66 of Department 4, 29 of Department 5. Ten respondents did not indicate their departments. The sample consisted of 251 females and 40 males, while six respondents did not indicate their gender. The average age of the respondents was 34,75 
$(\underline{\mathrm{SD}}=839)$ years. The race distribution in the obtained sample was: African $(\mathrm{N}=8)$, White $(\mathrm{N}=3)$, Asian $(\mathrm{N}=3)$ and Coloured $(\mathrm{N}=278)$. Seven respondents did not indicate to which race group they belong. In terms of qualifications: 15 Respondents had a Primary School qualification, 159 respondents had a qualification of between Grade 8 to Grade 10; 89 respondents had Grade 12; 20 respondents had a Tertiary qualification, of which two respondents had a degree. Twelve respondents did not indicate their qualifications. The respondents represented the following occupations: 222 shop floor employees, 30 clerks, 29 supervisors, 14 heads of departments and two senior managers. The respondents had an average of $11,3(\underline{\mathrm{SD}}=$ $7,3)$ years service to the company of which they have been reporting an average of $5,8(\underline{\mathrm{SD}}=5.14)$ years to their current supervisor or line manager.

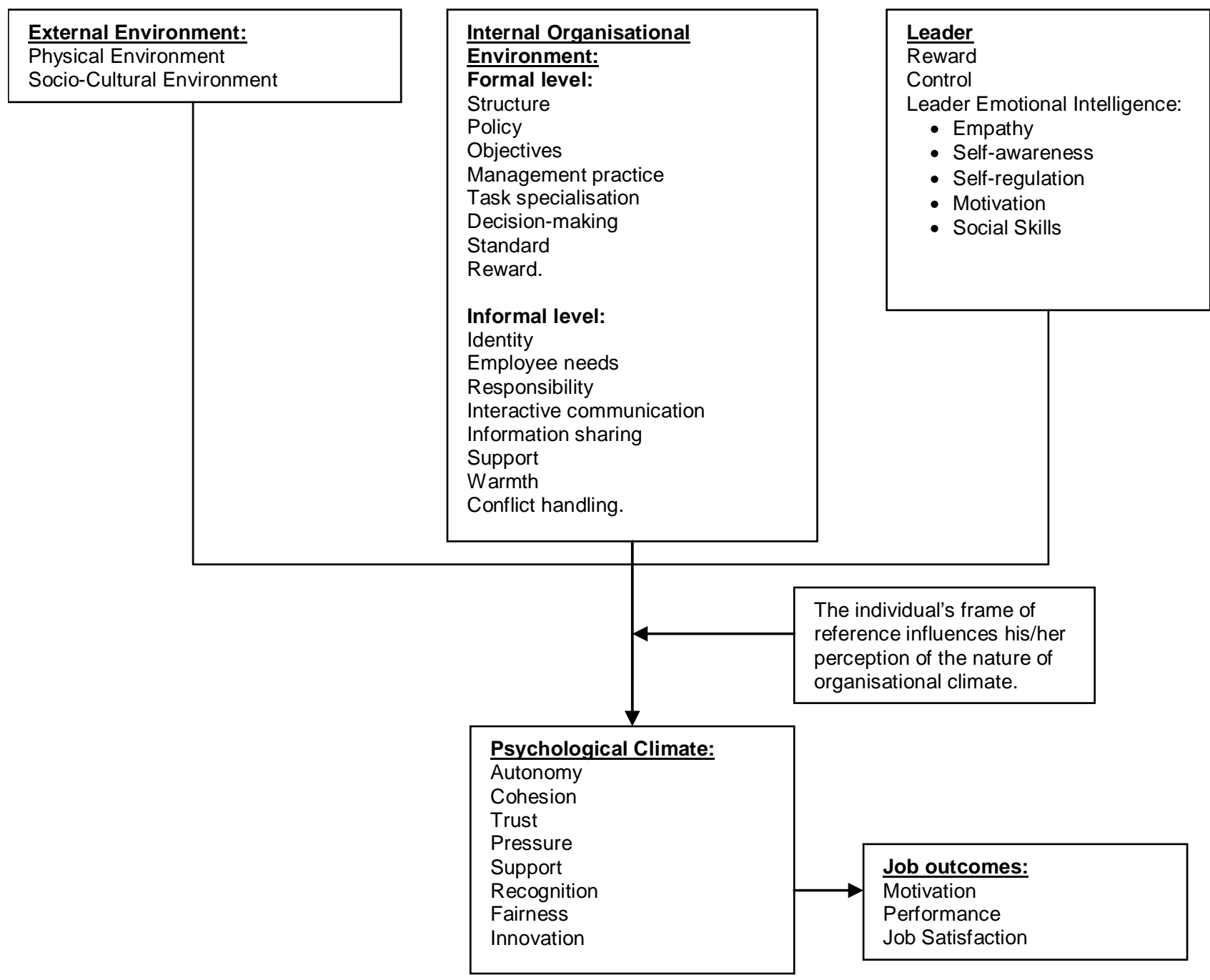

Figure 1: An integrated model of the antecedents of psychological climate (Adapted from Field \& Abelson, 1982: 183)

\section{Measuring instruments}

The constructs which were the focus of this study, were assessed or measured using the following measuring instruments.

\section{Emotional intelligence}

Emotional Intelligence was measured using the Swinburne University Emotional Intelligence Test (SUEIT) that was developed by the Organisational Psychology Research Unit of the University of Swinburne in Australia. The SUEIT was developed as a result of the search for answers to what the most definitive dimensions of the EI construct could be, based on this great plethora of different models and measures of emotional intelligence available.

In Australia, a large factor analytic study with a representative sample of the general population $(\underline{N}=310)$ was done. Most of the currently available measures of emotional intelligence were included in this battery. These included: 1) Mayer, Salovey, Caruso Emotional Intelligence test (MSCEIT) by Mayer, Salovey \& Caruso, (1999); 2) Bar-On Emotional Quotient Inventory (Bar-On, 1997); 3) Trait Meta-Mood Scale (Salovey, Mayer, Goldman, Turvey \& Palfai, 1995); 4) twenty-item Toronto Alexithymia ScaleII (TAS-20; Bagby, Taylor \& Parker, 1994); 5) the scale by Schutte et al. (1998) and 6) the scale by Tett, Wang, 
Thomas, Griebler \& Linkovich (1997). Each scale was factor analysed separately. The component score coefficients were used to form factor-based scores for each of the dimensions identified for each test. These dimensions were again used as "items" for the principle component analysis. This resulted in five factors having eigenvalues greater than one, thus matching the criteria and explaining $58 \%$ of the total variance. The following five factors were identified from the items: 1) Emotional recognition and expression, 2) Understanding others' emotions, 3) Emotions direct cognition, 4) Emotional management and 5) Emotional control. This empirically-based model of emotional intelligence, consisting of 64 items, is unidimensional, which means that the factors represent a set of related abilities concerned with how effectively emotions are dealt with in the workplace. The overall scale reliability (the standardised Cronbach alpha) of the questionnaire was found to be 0,88 while the alphas for the sub-scales were found to be: 1) Emotional recognition and expression: $\alpha=$ $0,73 ; 2)$ Understanding of emotions external: $\alpha=0,83 ; 3$ ) Emotions direct cognition: $\alpha=0,63$; 4) Emotional Management: $\alpha=0,72$ and 5) Emotional control: $\alpha=0,72$. The full-scale reliability and most sub-scales are high with the exception of the Emotions direct cognition sub-scale (SUIET), (Palmer \& Stough, 2002).

The $360^{\circ}$ version of the SUIET was used for this study. The participants had to respond to the 64 items on a six-point Likert-type scale $(1=$ never, $2=$ seldom, $3=$ sometimes, $4=$ usually, $5=$ always and $6=$ not sure). They were asked to indicate the extent to which the statements (i.e. items) are true of the way the person (i.e. their supervisor or line manager), whom they have been asked to rate, typically thinks, feels and deals with emotions at work. Some items are negatively worded and the scores on these items were reversed.

\section{Psychological climate}

Psychological climate was measured with the Organisational Climate Questionnaire (OCQ) developed by Koys and DeCotiis (1991). They defined psychological climate as “...an experiential-based, multi-dimensional, and enduring perceptual phenomenon, which is widely shared by the members of a given organisational unit. Its primary function is to cue and shape individual behaviour towards the modes of behaviour dictated by organisational demands" (Koys and DeCotiis, 1991: 266).

Koys and DeCotiis (1991) initially identified a list of over 80 organisational climate dimensions. Several decision rules were established to reduce the number of dimensions to a manageable and yet comprehensive universe of psychological climate dimensions. Firstly, selected dimensions had to be measures of perception as opposed to objective measures of climate. Secondly, selected dimensions were required to be descriptive, and not evaluative of the activities in question. Finally, dimensions were required not to be an aspect of organisational or task structure. Applying the above criteria resulted in the elimination of all objective, evaluative, affective and organisational structure measures. Sixty-one of the reported climate dimensions remained. These dimensions were subjected to further elimination by cluster analysis. The process resulted in forty-five dimensions being retained and categorised into eight concepts that are viewed as representing the psychological climate construct. The eight concepts were: 1) Autonomy, 2) Cohesion, 3) Trust, 4) Pressure, 5) Support, 6) Recognition, 7) Fairness and 8) Innovation.

Participants responded to a total of 40 items of the OCQ on a 7-point Likert-type scale ranging from "strongly disagree" to "strongly agree". Adding the scores of the relevant items in each dimension and dividing these totals by the number of items, measuring each dimension, determined the eight dimension scores for each respondent. This resulted in the psychological climate profile for a respondent. Some items are negatively worded and were reverse scored. Research by Koys and DeCotiis (1991) reported satisfactory psychometric properties of the items and scales. The reliability coefficients (Cronbach Aalpha) of the eight dimensions for two standardisation sample groups were: 1) Autonomy $\alpha=0,83$ (Group 1) and 0,76 (Group 2); 2) Cohesion $\alpha=0,87$ (Group 1) and 0,82 (Group 2); 3) Trust $\alpha$ $=0,88$ (Group 1) and 0,87 (Group 2); 4) Pressure $\alpha=0,81$ (Group 1) and 0,57 (Group 2); 5) Support $\alpha=0,89$ (Group 1) and 0,90 (Group 2); 6) Recognition $\alpha=0,83$ (Group 1) and 0,84 (Group 2); 7) Fairness $\alpha=0,82$ (Group 1) and 0,82 (Group 2); and 8) Innovation $\alpha=0,80$ (Group 1) and 0,87 (Group 2) (Koys and DeCotiis, 1991)

\section{Social desirability}

For the past four decades researchers have been concerned with the 'distorted' way people respond to surveys and test items. The results of surveys that investigate attitudes, opinions and personalities can be influenced by the effect of social responsibility or 'faking good' as a variable. Crowne and Marlowe (1960) included some of these items in surveys, in an attempt to assess and estimate the amount of variability that can be attributed to social desirability. This proportion of variance can be determined by following multiple regression procedures and then correcting the distortion by using partial correlation or canonical correlation analysis (Fraboni \& Cooper, 1989).

According to Geher, Warner and Brown (2001) the implementation of social-desirability scales have been shown to increase the reliability of self-report scales. Strahan and Gerbasi (1972) developed the 10-item unidimensional Marlowe-Crowne Scale-Short Form. It is based on the original model and scale of social desirability by Crowne and Marlowe (1960). An alpha coefficient of 0,70 was reported by Fraboni and Cooper (1989) for the scale.

\section{Data collection}

The 600 participants, drawn by means of random proportional cluster sampling, received a composite questionnaire that consisted of the following: a covering letter, a biographical section and the three measuring instruments. The covering letter briefly explained the reason for the survey, how to complete the questionnaires and whom to return it to. Complete confidentiality and 
anonymity was guaranteed. The three instruments were translated from English into Afrikaans by using the back translation method. Following this method, the text was translated into Afrikaans and then back to English. This was done so that the Afrikaans version could be regarded as equivalent to the English form and that results from the Afrikaans and English speakers would be comparable. Respondents were asked to evaluate the psychological climate, the perceived emotional intelligence of their supervisor/line-manager and completed the social desirability scale.

\section{Research design and statistical analysis}

The study can be described as quantitative and having a cross-sectional survey design. The following statistical analyses were done using SPSS Version 11: Exploratory Factor Analysis, Cronbach alpha, Pearson correlation coefficient, One-way ANOVA, Stepwise discriminant analysis and Hierarchical Multiple Regression analysis.

\section{Results}

The responses to the 64 items of the SUEIT and to the 40 items of the Organisational Climate Questionnaire were firstly subjected to Exploratory Factor Analysis (EFA) using SPSS (ver.11). The aim of the EFA was to identify a set of variables or factors that accounted for a major portion of the total variance of the original items. EFA is considered to indicate construct validity. The EFA was conducted using the Principal-axis factor extraction method, followed by Direct Oblimin rotation. The factor loadings in the rotated matrix were inspected after the factor structure was determined. An item was rejected if it had a loading of $\leq$ 0,30 on all factors or when it cross-loaded, i.e. if the loadings differed by $\leq 0,25$ across factors. The EFA was repeated and all items were rejected that did not comply with the above criteria, until a "clean" factor structure was obtained. The Kaiser criterion, which specifies that only factors with eigenvalues of 1.00 or greater should be retained and the so-called Scree test, was used as a guide to determine the number of factors to extract. Following this, the scale reliability was assessed using Cronbach alphas.

\section{Emotional intelligence}

The first round of EFA of the SUIET was performed using all of the 297 responses to the 64 items. Several rounds of EFA's specifying a three-factor solution eliminated the following number of items: Round $1-5$ items; Round $2-$ 17 items; Round $3-3$ items; and Round $4-1$ item. The final factor structure therefore contained 38 items. The EFA yielded three factors with eigenvalues exceeding 1: Factor 1: eigenvalue $=10,12$, explaining $20,65 \%$ of the total variance; Factor 2: eigenvalue $=3,86$ explaining $7,87 \%$ of the total variance; Factor 3: eigenvalue $=1,97$, explaining $4,01 \%$ of the total variance. The three factors together explained $32,53 \%$ of the total variance.

The Cronbach Alpha coefficients was found to be satisfactory for the scale $(\alpha=0,87)$, as well as for the factors (Factor 1: $\alpha=0.87$, Factor 2: $\alpha=0,79$ and Factor 3: $\alpha=$ $0,70)$.
After inspecting the items that loaded meaningfully, the three factors were identified as: EI factor $1=$ perception of and control over emotions, EI factor 2 = displaying emotions, and EI factor 3 = giving credence to emotions. This EFA-derived scale was considered to be a reliable (i.e. Cronbach alphas were all $>0,70$ and therefore satisfactory) and valid measure of EI (as based on the EFA results). The EFA-derived measurement model obtained from the EFA was used in subsequent analyses as a valid and reliable measure of emotional intelligence.

\section{Psychological climate}

After the first round of EFA on the responses to the Psychological Climate Questionnaire was performed (i.e. the responses to the 40 items of 297 participants) 11 items were removed as they did not meet the inclusion criteria. After the second round of EFA three items were removed, leaving 32 items in the final factor structure. The EFA revealed the presence of five meaningful factors with eigenvalues exceeding 1: Factor 1: eigenvalue $=11,25$, explaining $35,16 \%$ of the variance; Factor 2: eigenvalue = 2,82 , explaining $8,82 \%$ of the variance; Factor 3 : eigenvalue $=2,40$, explaining $7,51 \%$ of the variance; Factor 4 : eigenvalue $=1,90$, explaining $5,95 \%$ of the variance and Factor 5: eigenvalue $=1,17$, explaining $3,67 \%$ of the variance. Together, the five factors explained $61,1 \%$ of the variance. The Cronbach alpha coefficient for the EFAderived scale was found to be 0,93 and for the factors: Factor 1: $\alpha=0,94$, Factor 2: $\alpha=0,85$, Factor 3: $\alpha=0,82$, Factor 4: $\alpha=0,66$ and Factor 5: $\alpha=0,88$.

After inspecting the items that loaded meaningfully, the five factors were identified as: climate factor $1=$ Trust; climate factor 2 = Cohesion; climate factor $3=$ Autonomy; climate factor $4=$ Pressure and climate factor $5=$ Innovation. The EFA-derived measurement model obtained from the EFA was used in subsequent analyses as a valid and reliable measure of psychological climate.

\section{Social desirability}

The Pearson correlation coefficient was used to investigate the influence of social desirability on the responses of the participants. Only one small significant correlation between Social desirability and Trust $(\mathrm{r}=0,163, \mathrm{p}<0,01)$ was found. None of the other factors of psychological climate or emotional intelligence correlated significantly with social reliability.

\section{Analysis of variance between and within groups}

One-way ANOVA was used to determine whether the scores of members from the five departments on the dependant variable, psychological climate, differed from each other. The hypothesis being tested was that a single psychological climate profile exists in the organisation. There was a statistically significant difference at the $p<0,05$ level for scores for the departments on Trust $[\mathrm{F}(4$, $282)=2,62, \mathrm{p}=0,035]$, Autonomy $[\mathrm{F}(4,281)=4,23, \mathrm{p}=0,002]$, Pressure $[\mathrm{F}(4,281)=4,97, \mathrm{p}=0,001]$, and Total Psychological Climate $[F(4,281)=4,25, p=0,002]$. There was no 
significant difference between the departments for Cohesion and Innovation. The results are summarised in Table 3.

Having received a statistically significant difference in the overall ANOVA, post-hoc tests were carried out. Post-hoc comparisons using the Tukey HSD test indicated that the mean score for Department $1(\underline{\mathrm{M}}=4,66, \underline{\mathrm{SD}}=1,77)$ and Department $4 \quad(\underline{M}=4,85, \quad \underline{S D}=1,74)$ was significantly different from that of Department $5(\underline{\mathrm{M}}=5,85, \underline{\mathrm{SD}}=1,46)$ on Trust; Department $1(\underline{\mathrm{M}}=3,90, \underline{\mathrm{SD}}=1,87)$ and Department 2
$(\underline{\mathrm{M}}=4,03, \underline{\mathrm{SD}}=1,40)$ significantly differed from Department 5 on Autonomy; Department $1 \quad(\underline{\mathrm{M}}=3,1, \underline{\mathrm{SD}}=1,54)$ and Department $4(\underline{\mathrm{M}}=3,17, \underline{\mathrm{SD}}=1,62)$ significantly differed from Department $5(\underline{M}=4,53, \underline{S D}=1,53)$ on Pressure and on the Total Psychological Climate score, Department 1 $(\underline{\mathrm{M}}=4,33, \underline{\mathrm{SD}}=1,30)$, Department $2 \quad(\underline{\mathrm{M}}=4,52, \underline{\mathrm{SD}}=1,018)$, Department $3(\underline{\mathrm{M}}=4,62, \underline{\mathrm{SD}}=1,24)$ and Department 4 $(\underline{\mathrm{M}}=4,54, \underline{\mathrm{SD}}=1,24)$ significantly differed from Department $5(\underline{\mathrm{M}}=5,41, \underline{\mathrm{SD}}=1,15)$.

Table 3: Difference between departments (Oneway Anova)

\begin{tabular}{|c|c|c|c|c|c|}
\hline & Sum of Squares & df & Mean Square & $\mathbf{F}$ & Sig \\
\hline \multicolumn{6}{|l|}{ Trust: } \\
\hline Between groups & 28,330 & 4 & 7,083 & 2,622 & 0,035 \\
\hline Within groups & 761,843 & 282 & 2,702 & & \\
\hline Total & 790,173 & 286 & & & \\
\hline \multicolumn{6}{|l|}{ Cohesion: } \\
\hline Between groups & 9,082 & 4 & 2,271 & 0,876 & 0,479 \\
\hline Within groups & 730,951 & 282 & 2,592 & & \\
\hline Total & 740,033 & 286 & & & \\
\hline \multicolumn{6}{|l|}{ Autonomy: } \\
\hline Between groups & 44,111 & 4 & 11,028 & 4,227 & 0,002 \\
\hline Within groups & 735,739 & 282 & 2,609 & & \\
\hline Total & 779,849 & 286 & & & \\
\hline \multicolumn{6}{|l|}{ Pressure: } \\
\hline Between groups & 49,862 & 4 & 12,466 & 4,970 & 0,001 \\
\hline Within groups & 707,341 & 282 & 2,508 & & \\
\hline Total & 757,203 & 286 & & & \\
\hline \multicolumn{6}{|l|}{ Innovation: } \\
\hline Between groups & 21,974 & 4 & 5,493 & 1,815 & 0,126 \\
\hline Within groups & 850,713 & 281 & 3,027 & & \\
\hline Total & 872,686 & 285 & & & \\
\hline \multicolumn{6}{|c|}{ Total Psychological Climate: } \\
\hline Between groups & 22,790 & 4 & 5,698 & 4,245 & 0,002 \\
\hline Within groups & 377,122 & 281 & 1,342 & & \\
\hline Total & 399,912 & 285 & & & \\
\hline
\end{tabular}

\section{Stepwise discriminant analysis}

Due to the fact that the ANOVA is not robust against large numbers in groups to be compared (as was the case in the present study), discriminant analysis was used to predict department membership by means of psychological climate dimension's scores. This was done to determine whether a single psychological climate existed in the organisation. Discriminant analysis combines the independent variables into a single new variable called a discriminant function. According to this variable, participants' scores were distinguished or discriminated among those participants in the different categories of the dependent variable (Kinnear \& Gray, 2000). Wilk's Lambda was used to test the value of the discriminant function that was producing significant differences among the groups. A value between 0,8 and 1 was accepted as indicating a poor grouping. The scores on the psychological climate sub-scales were used as independent and departmental membership of respondents, as the dependant variable.

Table 6 provides an indication of the success rate of predictions of membership of the different departments. Correct classification of $75 \%$ and higher was considered to be a meaningful discrimination level. The overall success rate of classification was $29,4 \%$, which is low. Department 5 was the most accurately classified with $58,6 \%$ of the cases correct and Department 3 the lowest, with $16,3 \%$ of its members correctly classified. The Wilk's Lambda values varied between 0,861 and 0,997 , indicating poor grouping (results are shown in Table 4). 
Table 4: Differences between groups (Stepwise Discriminant Analysis)

\begin{tabular}{lcccccc}
\hline & \multicolumn{7}{c}{ \% Predicted group membership } & \\
\hline & Dept 1 & Dept 2 & Dept 3 & Dept 4 & Dept 5 & Total \\
Dept 1 & 40,0 & 15,6 & 4,4 & 20,0 & 20,0 & 100,0 \\
Dept 2 & 26,2 & 21,3 & 8,2 & 21,3 & 23,0 & 100,0 \\
Dept 3 & 10,5 & 17,4 & 16,3 & 26,7 & 29,1 & 100,0 \\
Dept 4 & 26,2 & 12,3 & 6,2 & 33,8 & 21,5 & 100,0 \\
Dept 5 & 13,8 & 6,9 & 6,9 & 13,8 & 58,6 & 100,0 \\
Ungrouped cases & 20,0 &, 0 & 10,0 & 20,0 & 50,0 & 100,0 \\
\hline
\end{tabular}

a. $29.4 \%$ of original grouped cases correctly classified

\begin{tabular}{lc}
\hline \multicolumn{1}{c}{ Test of function(s) } & Wilks' Lambda \\
\hline 1 through 4 &, 861 \\
2 through 4 &, 954 \\
3 through 4 &, 988 \\
4 &, 997 \\
\hline
\end{tabular}

It would seem as if group membership could not be predicted well by means of psychological climate scores. The groups were therefore considered not to be significantly different in terms of the scores on the OCQ. Based on this outcome, Hypothesis 1 is accepted.

\section{Pearson correlation coefficient}

The hypothesis that a significant positive relationship exists between leader emotional intelligence and psychological climate was investigated by means of the calculation of Pearson correlation coefficients. The Pearson correlation coefficient was used to determine the strength and direction of the relationship. Cohen (1988) suggests the following guidelines to qualitatively interpret the values obtained: 1) Small $(\mathrm{r}=+/-0,10$ to $+/-0,29), 2)$ Medium $(\mathrm{r}=+/-0,30$ to $+/-$ $0,49)$ and 3$)$ Large $(r=+/-0,50$ to $+/-1,00)$.

The correlation results can be seen in Table 5. Small significant correlations were found between a number of the emotional intelligence and psychological climate factors. There were medium level significant correlations between Emotional Intelligence Factor 1 (i.e. perception of and control over emotions) and Pressure $(\mathrm{r}=0,318, \mathrm{p}<0,01)$, Emotional Intelligence Factor 3 (i.e. giving credence to emotions $)$ and Trust $(\mathrm{r}=0,370, \quad \mathrm{p}<0,01)$, Emotional Intelligence Factor 3 and Innovation $(r=0,375, p<0,01)$, Emotional Intelligence Factor 3 and the Total Psychological Climate score $(r=0,418, p<0,01)$ and the Total Emotional Intelligence Factor and Trust $(r=0,376, p<0,01)$. Medium level significant correlations were also found between Total Psychological Climate and Total Emotional Intelligence $(\mathrm{r}=0,366, \mathrm{p}<0,01)$. The coefficient of determination $\left(\mathrm{r}^{2} \mathrm{x}\right.$ $100)$ indicates the percentage of variance that is shared between two variables. These were: $10,11 \%$ for Emotional Intelligence Factor 1 and Pressure; 13,7\% for Emotional Intelligence Factor 3 and Trust; $14,06 \%$ for Emotional Intelligence Factor 3 and Innovation; 17,5\% for Emotional Intelligence Factor 3 and the Total Psychological Climate score; and $14,14 \%$ for the Total Emotional Intelligence Factor and Trust.

Based on the results obtained, it is believed that Hypothesis 2 could be accepted.

\section{Hierarchical multiple regression analysis}

Hierarchical multiple regression analysis was used to further determine the relative contribution of each of the sub-factors of leader emotional intelligence to the prediction of scores on the psychological climate dimensions. The three dimensions of emotional intelligence were regressed against the five dimensions of psychological climate. By using Hierarchical Multiple Regression the variables were entered into blocks in a predetermined order. Age, number of years service and number of years worked under the supervisor or line-manager were entered into the first block (Model 1). During the second step the other independent variables were entered into a block (Model 2), thus statistically removing the possible effect of age, number of years service and number of years worked under the supervisor or linemanager. This would also be an indication of whether the independent variable, emotional intelligence, is able to explain some of the remaining variance in the dependent variable, psychological climate. The results are show in Table 6. 
Table 5: Pearson correlations coefficients

\begin{tabular}{|c|c|c|c|c|c|c|c|}
\hline & & Trust & Cohesion & Autonomy & Pressure & Innovation & Total Psych Climate \\
\hline $\begin{array}{l}\text { Perception of and } \\
\text { control over } \\
\text { emotions (EI1) }\end{array}$ & $\begin{array}{l}r \\
r^{2} \times 100\end{array}$ & $\begin{array}{l}.294 * * \\
8.64 \%\end{array}$ & .112 & $\begin{array}{l}.124 * \\
1.54 \%\end{array}$ & $\begin{array}{l}.318^{* *} \\
10.11 \%\end{array}$ & $\begin{array}{l}.116^{*} \\
1.35 \%\end{array}$ & $\begin{array}{l}.291 * * \\
8.47 \%\end{array}$ \\
\hline $\begin{array}{l}\text { Displaying emotions } \\
\text { (EI2) }\end{array}$ & $\begin{array}{l}r \\
r^{2} \times 100\end{array}$ & $\begin{array}{l}.220 * * \\
4.84 \%\end{array}$ & -.004 & .053 & .059 & $\begin{array}{l}.180 * * \\
3.24 \%\end{array}$ & $\begin{array}{l}.185^{* *} \\
3.42 \%\end{array}$ \\
\hline $\begin{array}{l}\text { Giving credence to } \\
\text { emotions (EI3) }\end{array}$ & $\begin{array}{l}r \\
r^{2} \times 100\end{array}$ & $\begin{array}{l}.370 * * \\
13.69 \%\end{array}$ & $\begin{array}{l}.242 * * \\
5.86 \%\end{array}$ & $\begin{array}{c}.247 * * \\
6.1 \%\end{array}$ & .097 & $\begin{array}{l}.375^{* *} \\
14.06 \%\end{array}$ & $\begin{array}{l}.418 * * \\
17.47 \%\end{array}$ \\
\hline Total EI & $\begin{array}{l}r \\
r^{2} \times 100\end{array}$ & $\begin{array}{c}.376 * * \\
14.14 \%\end{array}$ & $\begin{array}{l}.125 * \\
1.56 \%\end{array}$ & $\begin{array}{l}.160 * * \\
2.56 \%\end{array}$ & $\begin{array}{l}.265 * * \\
7.02 \%\end{array}$ & $\begin{array}{l}.230 * * \\
5.29 \%\end{array}$ & $\begin{array}{l}.366^{* *} \\
13.4 \%\end{array}$ \\
\hline
\end{tabular}

** Correlation is significant at the 0.01 level (2-tailed). * Correlation is significant at the 0.05 level (2-tailed).

Table 6: Hierarchical multiple regression: Model summary and standardised beta coefficients

\begin{tabular}{|c|c|c|c|c|c|c|c|c|c|c|c|c|c|c|c|c|c|}
\hline \multirow[b]{2}{*}{ 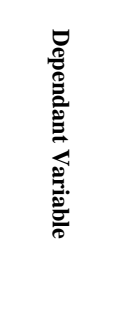 } & \multirow[b]{2}{*}{$\begin{array}{l}3 \\
\frac{2}{0} \\
\frac{0}{0}\end{array}$} & \multirow[b]{2}{*}{$\mathbb{N}_{N}$} & \multirow[b]{2}{*}{ 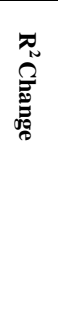 } & \multirow[b]{2}{*}{$\stackrel{1}{\Xi}$} & \multirow[b]{2}{*}{ 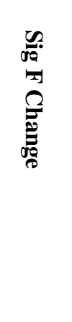 } & \multicolumn{12}{|c|}{ Standardised Coefficients: Beta } \\
\hline & & & & & & $\underset{d p}{\text { 品 }}$ & 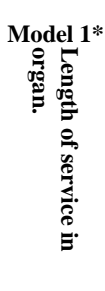 & 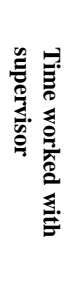 & 趸 & 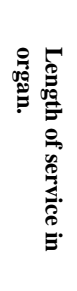 & 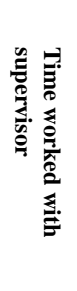 & 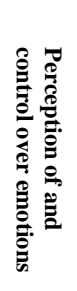 & 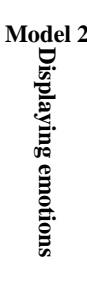 & 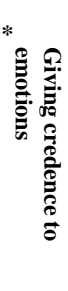 & 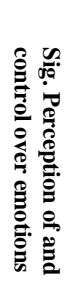 & 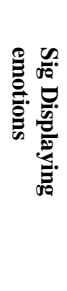 & 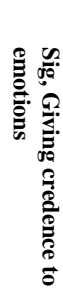 \\
\hline Trust & $\begin{array}{l}1 \\
2\end{array}$ & $\begin{array}{l}013 \\
202\end{array}$ & $\begin{array}{l}013 \\
190\end{array}$ & $\begin{array}{c}658(3) \\
1189 \\
(3)\end{array}$ & $\begin{array}{l}579 \\
000\end{array}$ & & & & & & & & & & & & \\
\hline Cohesion & $\begin{array}{l}1 \\
2\end{array}$ & $\begin{array}{l}019 \\
090\end{array}$ & $\begin{array}{l}019 \\
072\end{array}$ & $\begin{array}{c}972(3) \\
3937 \\
(3)\end{array}$ & $\begin{array}{l}408 \\
010\end{array}$ & 005 & -117 & 005 & -062 & -046 & -035 & 206 & 047 & 327 & 008 & 563 & 000 \\
\hline Autonomy & $\begin{array}{l}1 \\
2\end{array}$ & $\begin{array}{l}009 \\
074\end{array}$ & $\begin{array}{l}009 \\
065\end{array}$ & $\begin{array}{l}462(3) \\
35(3)\end{array}$ & $\begin{array}{l}709 \\
017\end{array}$ & 065 & 095 & -015 & 007 & 142 & -063 & 0094 & -107 & 263 & 252 & 214 & 003 \\
\hline Pressure & $\begin{array}{l}1 \\
2\end{array}$ & $\begin{array}{l}002 \\
105\end{array}$ & $\begin{array}{l}002 \\
103\end{array}$ & $\begin{array}{r}110(3) \\
575(3)\end{array}$ & $\begin{array}{l}954 \\
001\end{array}$ & 093 & 000 & 005 & 042 & 046 & -034 & 091 & -046 & 241 & 273 & 598 & 006 \\
\hline Innovation & 1 & 013 & 013 & $667(3)$ & 573 & 042 & -019 & -032 & 034 & 005 & -029 & 320 & -040 & 044 & 000 & 635 & 611 \\
\hline Tot Psy & 2 & 149 & 136 & $\begin{array}{c}7794 \\
(3) \\
179(3)\end{array}$ & 0911 & 066 & -124 & 101 & -005 & -059 & 053 & 029 & 061 & 344 & 718 & 466 & 000 \\
\hline & 2 & 216 & 213 & $\begin{array}{c}1286 \\
(3)\end{array}$ & 0000 & 064 & -005 & -019 & -042 & 099 & -019 & 0286 & -004 & 324 & 000 & 961 & 000 \\
\hline
\end{tabular}

** Model 2: Predictors: (Constant), Period of time working under supervisor/line manager - years, Age, Length of service in organisation, EQ Factor 1, EQ Factor 2, EQ Factor

After the variables in Block 1 (i.e. age, number of years service and number of years worked under the supervisor or line-manager) were entered, the overall model explained $0,4 \%$ of the variance of Total Psychological Climate. This model did not significantly predict the dependant variable $(\mathrm{p}>0.05)$. When Block 2 was entered, the model as a whole (i.e. age, number of years service and number of years worked under the supervisor or line-manager, as well as the emotional intelligence dimensions) explained $21,6 \%$ of the variance of Total Psychological Climate. The R Square change indicates that emotional intelligence explains an additional $21,3 \%$ in variance for Total Psychological Climate when the effects of age, number of years service and number of years worked under the supervisor or linemanager variables are controlled for. This is a statistically significant contribution as indicated by the change in F- value and $\mathrm{p}<0.01$. The independent variables that made a significant unique contribution to the regression equation predicting Total Psychological Climate, were: EQ Factor 3 giving credence to emotions (Beta $=0,324$ ) and EQ Factor 1 - perception of and control over emotions (Beta $=0,286$ ). Considering the Beta coefficients EQ Factor 3 made the strongest unique contribution to the equation.

After the variables in Block 1 (age, number of years service and number of years worked under the supervisor or linemanager) were entered, the overall model explained $1,3 \%$ of the variance of Trust. This model did also not significantly predict the dependant variable Trust $(\mathrm{p}>0.05)$. When Block 2 was entered, the model as a whole (i.e. age, number of years service and number of years worked under the supervisor or line-manager, as well as the emotional 
intelligence dimensions) explained $20,2 \%$ of the variance of Trust. The $100 x \mathrm{R}$ Square change indicates that emotional intelligence explains an additional $19 \%$ of the variance in Trust when the age, number of years service and number of years worked under the supervisor or line-manager variables are controlled. This is a statistically significant contribution as indicated by the change in F-value $(p<0,01)$. The independent variables that made a significant contribution to the regression equation, were: EQ Factor 3 - giving credence to emotions $($ Beta $=0,327)$ and EQ Factor 1 - perception of and control over emotions (Beta $=0,206$ ). Considering the Beta coefficients EQ Factor 3 once again made the strongest unique contribution to the equation using Trust as the dependant variable.

After the variables in Block 1 (age, number of years service and number of years worked under the supervisor or linemanager) were entered, the overall model explained $1,9 \%$ of the variance of Cohesion. This model, as before did not significantly predict the dependant variable $(p>0.05)$. When Block 2 was entered, the model as a whole (age, number of years service and number of years worked under your supervisor or line-manager, as well as the emotional intelligence dimensions) explained $9 \%$ of the variance of Cohesion. The 100xR Square change indicates that emotional intelligence explains an additional $7,2 \%$ in variance in Cohesion when the age, number of years service and number of years worked under the supervisor or linemanager variables are controlled. This is a statistically significant contribution as indicated by the change in Fvalue $(p<0,01)$. The only independent variable that statistically and significantly contribute to the prediction of Cohesion was EQ Factor 3 - giving credence to emotions $($ Beta $=0,263)$.

After the variables in Block 1 (age, number of years service and number of years worked under the supervisor or linemanager) were entered, the overall model explained $0,9 \%$ of the variance of Autonomy. This model did not significantly predict the dependant variable $(p>0.05)$. When Block 2 was entered, the model as a whole (age, number of years service and number of years worked under the supervisor or linemanager, as well as the emotional intelligence dimensions) explained $7,4 \%$ of the variance of Autonomy. The $100 \mathrm{xR}$ Square change indicates that emotional intelligence explains an additional $6,5 \%$ in variance for Autonomy when the age, number of years service and number of years worked under the supervisor or line-manager variables are controlled. This is a statistically significant contribution as indicated by the change in F-value $(p=0,017)$. The only independent variable that statistically and significantly contribute to the explanation of emotional intelligence influencing Autonomy was EQ Factor 3 - giving credence to emotions (Beta $=$ $0,241)$.

After the variables in Block 1 (age, number of years service and number of years worked under the supervisor or linemanager) were entered, the overall model explained $0,2 \%$ of the variance of Pressure. This model, as before did not significantly predict the dependant variable $(p>0.05)$. When Block 2 was entered, the model as a whole (age, number of years service and number of years worked under the supervisor or line-manager, as well as the emotional intelligence dimensions) explained $10,5 \%$ of the variance in Pressure. The R Square change X 100 indicates that emotional intelligence explains an additional $10,3 \%$ in variance for Pressure when the age, number of years service and number of years worked under the supervisor or linemanager variables are controlled. This is a statistically significant contribution as indicated by the change in Fvalue $(p=0,001)$. The only independent variable that statistically and significantly contribute to the regression equation was EQ Factor 1 - perception of and control over emotions $($ Beta $=0,320)$.

After the variables in Block 1 (age, number of years service and number of years worked under the supervisor or linemanager) were entered, the overall model explained $1.3 \%$ of the variance of Innovation. When Block 2 was entered, the model as a whole (age, number of years service and number of years worked under the supervisor or line-manager, as well as the emotional intelligence dimensions) explained $14.9 \%$ of the variance of Innovation. The 100xR Square change indicates that emotional intelligence explains an additional $13.6 \%$ in variance for Innovation when the age, number of years service and number of years worked under the supervisor or line-manager variables are controlled. This is a statistically significant contribution as indicated by the change in F-value of 0,001 . The only independent variable that statistically and significantly contributed to predicting Pressure was: EQ Factor 3 - giving credence to emotions $($ Beta $=0,344)$.

\section{Discussion}

The participants are believed to have a sound knowledge of their company and their respective supervisors or line managers, based on the fact that have an average of 11,3 years service to the company, of which they have been reporting an average 5,8 years to their current supervisor or line managers. Based on this finding it would seem that they are in a position to meaningfully evaluate the psychological climate of the organisation and the perceived emotional intelligence of their supervisor or line-manager.

In this study, the Exploratory Factor Analysis provided support for only 3 of the 5 dimensions of the SUIET developed by Palmer and Stough (2002). Further, only five of the eight dimensions of the Organisational Climate Questionnaire of Koys and DeCotiis (1991) were replicated in this sample. A possible reason for the decrease of factors in both scales could be that the South African respondents understood and interpreted the items differently to those participants in Australia and the United States of America, respectively. An explanation for the different interpretations could be that the relatively low qualification level of the respondents that may not have met the minimum requirements of the scales. Based on Australian school levels the SUIET is specified to be used by eight to ninth grade reading level respondents. The highest average qualification level attained by the participants in the obtained sample was Grade 8 to Grade 10 or equivalent. This may be a lower education level as the prescribed requirement to comprehend and complete the test. 
The fact that the questionnaires were drafted in English, although also translated to Afrikaans, could also have contributed to a misinterpretation of the questions. English is the first language of only $14.8 \%$ of the participants. A further explanation for the change in the factor structure of the instrument could be the cultural difference in the interpretation of the items. It would also seem that the SUEIT is possibly not entirely robust against cultural differences. .

An important finding was that social desirability did not seem to have a significant influence on the participants' responses. Only one small significant correlation could be found between Social desirability and Trust $(r=0,163$, $\mathrm{p}<0,01$ ). No other significant correlations were found between social desirability and the factors of emotional intelligence and psychological climate. Social desirability was therefore not taken into account in the analysis of the data.

The statistically significant difference (at the $\mathrm{p}<0,05$ level) for psychological climate scores for the five departments $[\underline{F}(4,281)=4,25, \underline{p}=0,002]$, indicated that there may be a significant difference among the mean scores of psychological climate, for the five departments. Post-hoc comparisons using the Tukey HSD test indicated that the psychological climate in Department 1, 2, 3 and 4 differed significantly from Department 5. However, stepwise discriminant analysis indicated that the psychological climate factors did not meaningfully group participants into their departments well (the overall Wilk's Lambda value obtained is 0,861). Given that this may be the more appropriate technique to use in this sample, it lead one to believe that there was no significant difference in psychological climate among the different departments.

This study was an exploratory attempt to determine whether leader emotional intelligence is related to the psychological climate. The Pearson correlation and the Hierarchical Multiple Regression analysis provided support for the hypothesis that a significant positive relationship exists between leader emotional intelligence and psychological climate. The Pearson correlation coefficient for the relationship was found to be practically significant $(\mathrm{r}=0,366$, $\mathrm{p}<0,01$ which is $>0,3$ as described by Cohen (1998)). A Hierarchical Multiple Regression showed that none of the biographical information variables significantly influenced the dependent variable i.e. psychological climate. The Hierarchical Multiple Regression did show that two of the three factors of emotional intelligence significantly predicted psychological climate in the organisation. The third EQ factor, i.e. giving credence to emotions was consistently the best predictor of psychological climate and its dimensions. This result underlines the importance of giving authority and credibility to emotions within the dyadic relationship between leaders and followers within the organisational context.

\section{Limitations and recommendations for future research}

A limitation of this study, which plagues studies of this kind, is the presence of common method variance in the measures that affect the correlations between the independent and dependent variables. A further limitation is the nature of the sample - the study was based on a single organisation in the clothing manufacturing industry. Care should thus be taken not to generalise the findings to other organisations. The portability of the measuring instruments can be identified as another limitation as the instruments were developed in a culture that was very different than the one used in this study. These issues should be addressed as far as possible in future studies. Future studies should endeavour to better conceptualise this relationship and attempt to replicate these findings in other organisational contexts.

\section{Implications for management}

This study provided support for the view that organisations should carefully recruit, select and develop leaders as they have the ability to influence the psychological climate of an organisation, consequently impacting on the performance of the organisation. Adequate training and development for leaders that score low on emotional intelligence should be provided. It is speculated that leaders who have higher levels of emotional intelligence create stronger interpersonal relationships and trust with their subordinates, lead and support them more effectively, and function better under pressure. These characteristics are known to contribute to a successful organisation.

\section{References}

Bagby, R.M., Taylor, G.J. \& Parker, J.D.A. 1994. 'The Twenty-Item Toronto Alexithymia Scale II. Convergent, discriminate, and concurrent validity', Journal of Psychosomatic Research 38: 33-40.

Bar-On, R. 1997. Bar-On emotional quotient inventory (EQi): Technical manual. Toronto: Multi-Health Systems.

Boyatzis, R.E., Goleman, D. \& Hay/McBer. 1999. Emotional competence inventory. Boston: HayGroup.

Campbell, J., Dunnette, M.D., Lawler, E.E. \& Weick, K.E. 1970. Managerial behavior, performance, and effectiveness. New York: McGraw-Hill.

Carrol, J.B. 1993. Human cognitive abilities: A survey of factor-analytic studies. New York: Cambridge University Press.

Cilliers, F. \& Kossuth, S. 2002. 'The relationship between organisational climate and salutogenic functioning', $S A$ Journal of Industrial Psychology, 28(1):8 -13.

Cohen, J.W. 1988. Statistical power analysis for the behavioural sciences. $2^{\text {nd }}$ Edition. Hillsdale, NJ: Lawrence Erlbaum Associates.

Cooper, R.K. \& Sawaf, A. 1997. Executive EQ: Emotional intelligence in leadership and organisations. New York: The Berkley Publishing Group. 
Crowne, D.P. \& Marlowe, D. 1960. 'A new scale of social desirability independent of psycho-pathology', Journal of Consulting Psychology, 24: 349 - 354.

De Vries, M.F.R.K. 2001. The leadership mystique. Great Britain: Pearson Education.

Field, R.H.G. \& Abelson, M.A. 1982. 'Climate: A reconceptualisation and proposed model', Human Relations, 35(3):181 - 201.

Forehand, G.A. \& Gilmer, B. 1964. 'Environmental variation in studies of organisational behaviou', Psychological Bulletin, 62(6):361 - 382.

Fraboni, M. \& Cooper, D. 1989. 'Further validation of three short forms of the Marlowe-Crowne Scale of Social Desirability', Psychological Reports, 65:595 - 600.

Gardner, H. 1983. Frames of mind. New York: Basic Books.

Geher, G., Warner, R., \& Brown, A. 2001. 'Predictive ability of emotional accuracy research scale', Intelligence, 29:373 - 388 .

Glick, W.H. 1985. 'Conceptualising and measuring organisational and psychological climate: Pitfalls in multilevel research', Academy of Management Review, 10(3):601-616.

Goleman, D. 1995. Emotional intelligence: Why it matters more than IQ. New York: Bantam Books.

Goleman, D. 1998. Working with emotional intelligence. New York: Bantam Books.

Goleman, D. 2002. The new leaders: Transforming the art of leadership into the science of results. Great Britain: Little, Brown.

Guion, R.M. 1973. 'A note on organizational climate', Organizational Behavior and Human Performance, 9:120 125.

Hellriegel, D. \& Slocum, J.W. 1974. 'Organisational climate: Measures, research and contingencies', Academy of Management Journal, 17:255 - 280.

Hughes, R.L., Ginnet R.C. \& Curphy, G.J. 2002. Leadership: Enhancing the lessons of experience. $4^{\text {th }}$ Edition. New York: McGraw-Hill.

James, L.R. \& Jones, A.P. 1974. 'Organisational climate: A review of theory and research', Psychological Bulletin, 81(12):1096 - 1112 .

Joyce, W.F. \& Slocum, J. 1982. 'Climate discrepancy: Refining the concepts of psychological and organisational climate', Human Relations, 35(11):951 - 972.

Kelner, S.P, Rivers, C.A. \& O'Connell K.H. 1996. Managerial style as a behavioural predictor of organisational climate. Boston: McBer \& Company.
Kinnear, P.R. \& Gray, C.D. 2000. SPSS for Windows made simple. Release 10. Hove, East Sussex: Psychology Press Ltd.

Koys, D.J. \& DeCotiis, T.A. 1991. 'Inductive measures of psychological climate', Human Relations, 44(3):265 - 285.

Litwin, G.H. \& Stringer, R.A. 1968. Motivation and organisational climate. Boston: Division of Research, Graduate School of Business Administration, Harvard University.

Mayer, J.D. \& Salovey, P. 1997. 'What is emotional intelligence?' In Salovey, P. \& Sluyter, D. Emotional development and emotional intelligence: Educational implications. New York: Basic Books.

Mayer, J.D., Salovey, P. \& Caruso, D. 1999. Instruction Manual for the MSCEIT.

Mayer, J.D., Salovey, P. \& Caruso, D.R. 2000. 'Emotional intelligence as Zeitgeist, as personality, and as a mental ability'. In Bar-On, R \& Parker, J.D.A. (Eds.). The handbook of emotional intelligence. San Francisco: JosseyBass.

Moran, E.T. \& Volkwein, J.F. 1992. 'The cultural approach to the formation of organisational climate', Human Relations, 45 (1): 19 - 47.

Ostroff, C. \& Schmitt, N. 1993. 'Configurations of organisational effectiveness and efficiency', Academy of Management Journal, 36(6):1345 - 1357.

Palmer, B. \& Stough, C. 2002. Workplace SUEIT interim technical manual. Version 2. Swinburne: University of Technology.

Petrides, K.V. \& Furnham, A. 2000a. 'On the dimensional structure of emotional intelligence', Personality and individual differences, 29:313 - 320.

Petrides, K.V. \& Furnham, A. 2000b. 'Gender differences in measured and self-estimated trait emotional intelligence', Sex Roles, 42(5/6):449 - 461.

Salovey, P. \& Mayer, J.D. 1990. 'Emotional intelligence', Imagination, Cognition and Personality, 9(3):185 - 211.

Salovey, J. Mayer, S. Goldman, C. Turvey \& T. Palfai, 1995. Emotional attention, clarity and repair: Exploring emotional intelligence using the Trait Meta-Mood Scale. In: Pennebaker, J.W. (Ed.): Emotion, disclosure, and health. Washington, DC: American Psychological Association.

Schein, E. H. 1989. 'Organizational culture: What is it and how to change it'. In Evans, P., Doz, Y. \& Laurent, A. (eds.). Human resource management in international firms. MacMillan Press: London.

Schneider, B. 1973. 'The perception of organisational climate: The customer's view', Journal of applied Psychology, 57:248 - 256. 
Schneider, B. \& Reichers, A.E. 1983. 'On the etiology of climates’, Personnel Psychology, 36:19-39.

Scholtz, E.M. 2002. The impact of an intervention on the relationship between leadership characteristics and organisational climate in a mining environment. Unpublished master's thesis, University of Potchefstroom, Potchefstroom.

Schutte, N.S., Malouff, J.M., Hall, L.E., Haggerty, D.J., Cooper, J.T., Golden, C.J. \& Dornheim, L. 1998. 'Development and validation of a measure of emotional intelligence', Personality and Individual Differences, 25:167 -177 .

Sparrow, P.R. \& Gaston, K. 1996. 'Generic climate maps: A strategic application of climate survey data?', Journal of Organizational Behavior, 17:679 - 698.

Strahan, R. \& Gerbasi, K.C 1972. 'Short, homogeneous versions of the Marlowe-Crowne Socail Desirability Scale', Journal of Clinical Psychology, 28(2):191 - 193.

Tett, R., Wang, A., Thomas, M., Griebler, J., \& Linkovich, T. 1997. Testing a model of emotional intelligence'. Paper presented at the 1997 Annual Convention of the Southeastern Psychological Association, Atlanta, GA.

Tustin, C.M. 1993. 'A consensus approach to the measurement of organisational climate', Journal of Industrial Psychology, 19(1):1 - 4 .

Walker, R.E. \& Foley, J.M. 1973. 'Social intelligence: History and measurement', Psychological Reports, 33:839864.

West, W.A., Smith, H., Lu Feng, W. \& Lawthom, R. 1998. 'Research excellence and departmental climate in British universities', Journal of Occupational and Organisational Psychology, 71:261 - 281. 